\title{
Computer-Assisted Lead Placement for Peripheral Nerve Evaluation Test in a Candidate for Sacral Neuromodulation
}

\author{
Gianluca Sampogna ${ }^{1,2}$, Emanuele Montanari ${ }^{2}$, Michele Spinelli ${ }^{1}$ \\ ${ }^{1}$ Unit of Neurourology, ASST Grande Ospedale Metropolitano Niguarda, Milano, Italy \\ ${ }^{2}$ Unit of Urology, Fondazione IRCCS Ca’ Granda, Ospedale Maggiore Policlinico, Università degli Studi di Milano, Milano, Italy
}

Our aim was to report the first case of computer-assisted lead placement (CALP) for a peripheral nerve evaluation (PNE) test in a 55-year-old woman affected by chronic pelvic pain, who was a candidate for sacral neuromodulation (SNM). The first PNE test failed due to nonoptimal lead placement. We utilized a surgical navigation system (SNS) with electromagnetic tracking to guide the lead placement to the S3 right nerve roots. Neither intra- nor postoperative complications occurred. After 2 weeks, the patient reported $>50 \%$ symptom improvement, so she was recommended to receive a definitive SNM implant. Our case report demonstrated the feasibility and safety of CALP for the PNE test. Since the use of an SNS may guide easy and precise lead placement along the $\mathrm{S} 3$ afferent nerve roots, further studies are mandatory to outline the advantages and limits of this innovative technique.

Keywords: Sacral neuromodulation; Peripheral nerve evaluation; Pelvic pain; Computer-assisted surgery

- Research Ethics: The study was performed according to the Declaration of Helsinki and approved by the Institutional Review Board of ASST

Grande Ospedale Metropolitano Niguarda. The patient provided written consent for the procedure and case presentation.

- Conflict of Interest: No potential conflict of interest relevant to this article was reported.

Sacral neuromodulation (SNM) has represented a real revolution in functional urology over the last 25 years [1]. Thanks to its use and research into its mechanisms of action, we have achieved improved our knowledge about the visceral control of pelvic organs by the central nervous system [2]. SNM is, currently, a minimally invasive therapy consisting of mild electrical impulses to the sacral nerve roots (S2-4), which supply autonomic and somatic pelvic innervation, to modulate their activity. SNM is indicated for patients affected by overactive bladder, non-obstructive urinary retention, and chronic fecal incontinence. SNM has recently gained popularity as a treatment for chronic pelvic pain (CPP).

Since SNM is expensive and not optimal for all patients, a pe- ripheral nerve evaluation (PNE) test may be performed to predict the likelihood of success of a definitive implant [3]. The lead should be placed along the S3 afferent nerve roots under fluoroscopic and/or ultrasound guidance [4]. This procedure is performed by trial and error, while evaluating the patient's motor and/or sensory responses [5]. Thanks to technological advancements, the use of a novel image-guided surgical navigation system (SNS) may facilitate the procedure, helping surgeons during preoperative planning and providing intraoperative guidance [6].

We report the first case of computer-assisted lead placement (CALP) for the PNE test in a candidate for SNM.

Corresponding author: Michele Spinelli (D) https://orcid.org/0000-0002-6245-0205 Unit of Neurourology, ASST Grande Ospedale Metropolitano Niguarda, Piazza dellOspedale Maggiore 3, 20162 Milano, Italy

E-mail: michele.spinelli@ospedaleniguarda.it

Submitted: March 30, 2020 / Accepted after revision: July 22, 2020 


\section{CASE REPORT}

A 55-year-old woman was referred to our tertiary referral center for SNM. Five years previously she had undergone transobturator tape surgery for severe urinary incontinence. After 1 month, she underwent partial tape removal because of urethral erosion. Later, she complained of CPP. After 3 years she underwent complete tape removal because of new-onset urethral erosion, but CPP persisted with associated urge incontinence (1-2 pads/day). The pain was constant (numeric rating scale [NRS] $=3-4$ out of 10 ) and responded only to daily cannabis drops. She also started psychotherapy and took vortioxetine.

\section{Clinical Findings and Investigations}

Our visit revealed a minimal cystocele. The International Consultation on Incontinence Questionnaire-Short Form (ICIQSF) score was 11 out of 19. The International Cystitis Symptom and Problem Indexes (ICSI and ICPI) scores were, respectively, 11 out of 19 and 15 out of 16 . A bowel diary revealed regular intestinal function. The patient had not engaged in sexual intercourse over the last months. A videourodynamic study documented urgency and increasing pain for bladder filling of 150 $\mathrm{mL}$. A study of motor-evoked potentials from the pelvic floor highlighted a symmetrical bilateral response.

We proceeded with the PNE test, which was characterized by an unsuccessful lead placement to the left S3 foramen. With difficulty, we managed to place it contralaterally. After 2 weeks with the temporary lead, the patient reported no significant symptom improvement.

Since we were not sure whether the lead had been placed correctly, we decided to adopt an imaging-guided approach. We requested magnetic resonance imaging with a $1.5 \mathrm{~T}$ scanner, which showed a radicular cyst at the left S2 foramen (Fig. 1), so we planned to position the lead on the right, where the nerve course appeared physiological.

\section{Computer-Assisted Lead Placement}

The SNS required high-resolution radiological images, obtained through a mobile $\mathrm{x}$-ray system. The patient was in the prone position with 8 fiducial markers placed randomly over the sacrum (Fig. 2A).

As the SNS, we used StealthStation S7 (Medtronic, Dublin, Ireland), choosing the electromagnetic (EM) tracking option. We positioned the AxiEM (Medtronic, Dublin, Ireland) magnetic field emitter near the patient's sacrum and attached the

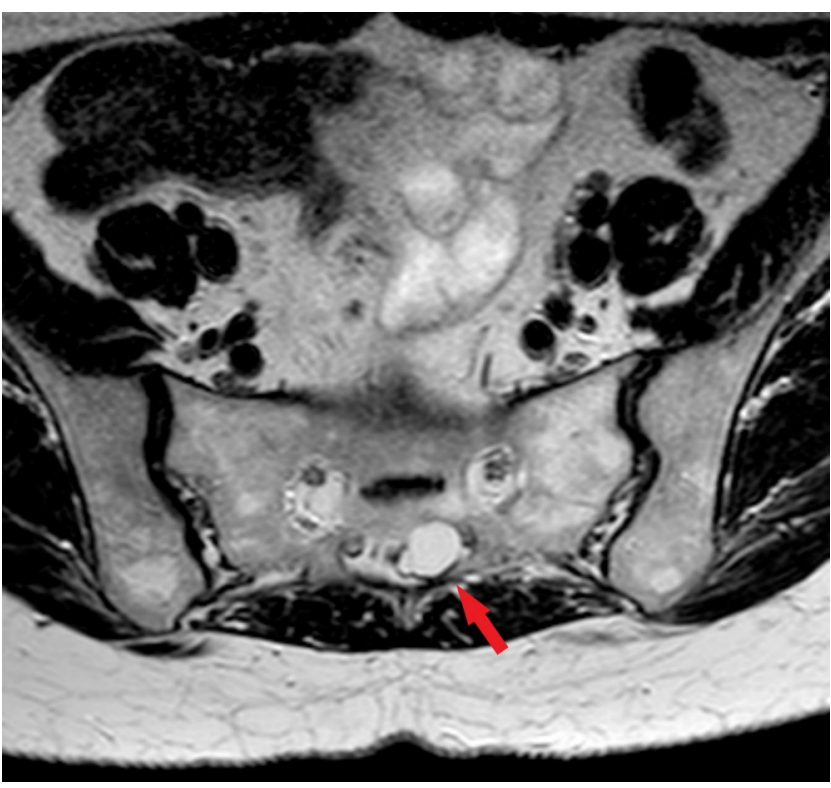

Fig. 1. T2-weighted turbo-spin-echo magnetic resonance imaging showing a 15-mm radicular cyst (arrow) at the left S2 foramen.

EM noninvasive patient tracker to the patient's left gluteus (Fig. 2B).

Firstly, we performed registration (i.e., the linkage of the physical space, or the area of surgical interest) with the image space obtained radiologically. The image-to-patient registration was performed by touching the fiducial markers with the AxiEM tracer pointer. The registration error metric (REM) was $1.6 \mathrm{~mm}$ with 7 fiducial markers; we excluded the most cranial fiducial because it increased the REM (Fig. 2C).

The obtained 3-dimensional model on the SNS screen was used for preoperative planning. We set the target point at the medial superior quarter of the right S3 foramen. We selected the insertion point on the skin to obtain a straight trajectory, avoiding obstacles and with a $60^{\circ}$ angle with the skin surface. The navigation was performed with the StealthStation EM flexible stylet.

We utilized a Basic Evaluation Kit (Medtronic) for the PNE test. Due to the need to use the stylet, instead of the standard lead insertion needle, we used a $5 \mathrm{~F}$ and $25-\mathrm{cm}$ ring drainage catheter (Cook Group, Bloomington, IN, USA), shortening it to a stylet length of $23 \mathrm{~cm}$ (Fig. 2D). We placed a Steri-Strip 23 $\mathrm{cm}$ from the lead tip to recognize when the tip reached the target point during the lead insertion.

With the patient under local anesthesia, we performed a skin incision at the insertion point, and advanced with the stylet in- 

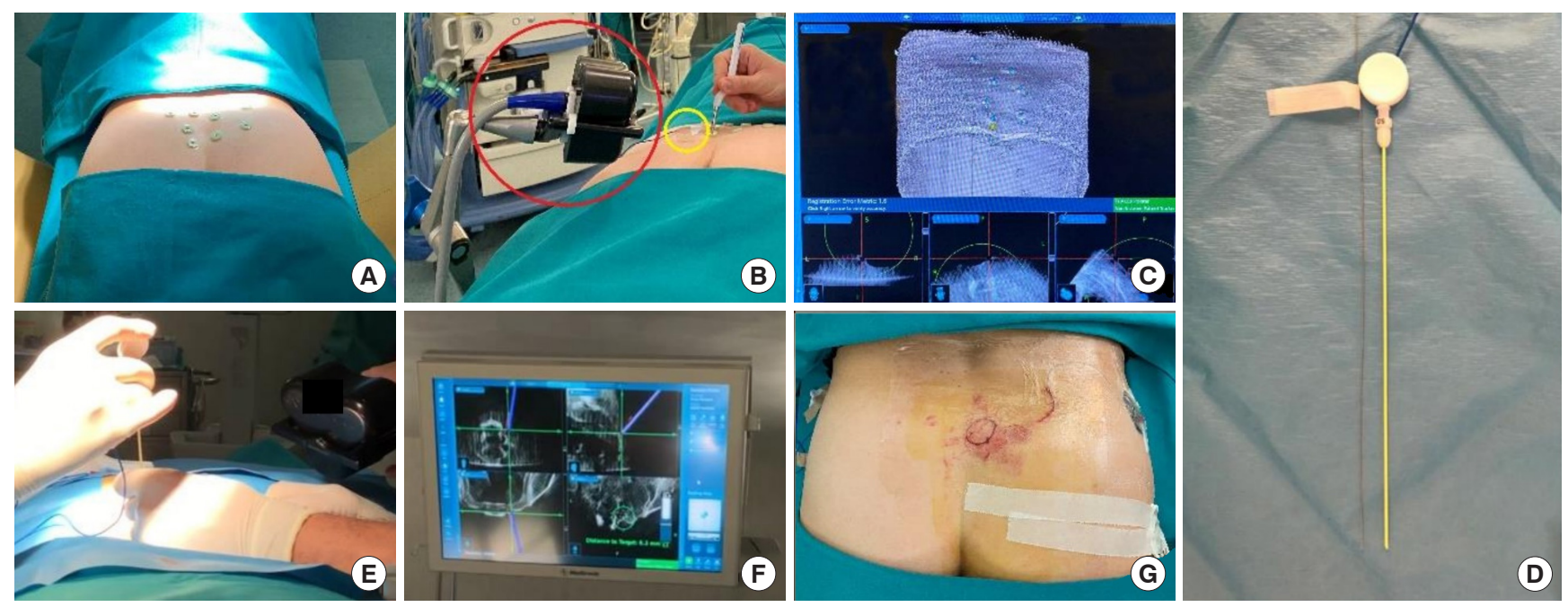

Fig. 2. Computer-assisted lead placement for the peripheral nerve evaluation (PNE) test. (A) The patient was prone. We obtained the radiological images for surgical navigation with a mobile x-ray system, after randomly placing 8 fiducial markers over the sacrum. (B) We used the StealthStation S7 Surgical Navigation System (Medtronic, Dublin, Ireland), with electromagnetic (EM) tracking, so we placed the AxiEM (Medtronic) magnetic field emitter (greater circle) near the patient's sacrum and attached the EM noninvasive patient tracker to the patient's left gluteus (smaller circle). We then touched all the fiducial markers with the tracer pointer to perform the image-to-patient registration (i.e., the linkage of the physical space, or the area of surgical interest, with the image space obtained radiologically). (C) The result of the image-to-patient registration was a 3-dimensional model on the navigation system screen for preoperative planning and intraoperative guidance. We obtained a valid registration error metric of $1.6 \mathrm{~mm}$ considering the 7 caudal fiducial markers and excluding the most cranial one. We set the target point at the medial superior quarter of the right S3 foramen, while we selected the insertion point on the skin to obtain a straight trajectory, avoiding obstacles and with a $60^{\circ}$ angle with the skin surface. (D) The lead used for the PNE test was shown on the left, while the sterile stylet for surgical navigation was inside a drainage catheter on the right. The insertion needle for the PNE test (not shown in the figure) was constructed to allow the release of the lead (diameter, $0.86 \mathrm{~mm}$; length, $30 \mathrm{~cm}$ ) and could not hold the stylet (diameter, $1.2 \mathrm{~mm}$; length, $23 \mathrm{~cm}$ ). Therefore, we used and shortened a 25-cm-long 5F drainage catheter with the tip-tracked stylet inside to allow the surgical navigation. When we reached the target point with the stylet tip, it was necessary to remove the stylet and insert the lead inside the drainage catheter. Therefore, we placed a SteriStrip on the lead $23 \mathrm{~cm}$ from the tip to recognize when the tip reached the target point during the lead insertion. (E) After local anesthesia with lidocaine, we performed a scalpel skin incision, inserted the drainage catheter with the navigation stylet inside and proceeded towards the target point following the indications showed on the screen. (F) The navigation system screen showed the stylet tip position inside the patient's sacral area, created digitally by radiological images. We followed the indicated trajectory towards the target point. In the lower right screen, the distance to the target point was reported in real time. The other 3 quadrants of the screen separately displayed real-time axial, sagittal, and coronal views of the active stylet trajectory. When we reached the target point (stylet tip to target point distance, $0.0 \mathrm{~mm}$ ), we removed the navigation stylet and inserted the lead inside the drainage catheter up to the marker placed previously. We confirmed the successful lead placement by evaluating the patient's electrical response. Then, we gently removed the drainage catheter, paying attention to leave the lead in place and avoid tip displacement from the target point. (G) Finally, the lead exited from the skin and, after an intradermal passage for fixation, was connected to the cable of an external neurostimulator.

side the catheter towards the target point, carefully following the trajectory established previously (Fig. 2E). The SNS screen showed axial, sagittal, and coronal views of the stylet position inside the patient's body and reported the distance to the target point in real time (Fig. 2F). When we reached the target point with the catheter distal tip, we extracted the stylet and inserted the lead up to the depth indicator positioned previously.

We used the Enhanced Verify (Medtronic) evaluation system to estimate the response. The patient reported a nonpainful stimulus as a pulling sensation in the rectum, extending forward to the external genitalia, with the following parameters: intensity, $0.9 \mathrm{~mA}$; frequency, $14 \mathrm{~Hz}$; pulse width, $210 \mu \mathrm{s}$. We retracted the catheter, paying attention to leave the lead in place and to avoid lead tip displacement. After catheter removal, we confirmed the optimal lead position with novel electrical stimuli. The lead was passed subcutaneously to secure it furtherly 
and connected to the patient cable with a ground pad (Fig. 2G).

The operative time was about 30 minutes. A transparent medication was applied. No intraoperative complications occurred.

\section{Postoperative Period}

On the first postoperative day, we attached the external neurostimulator, setting the parameters as tested the previous day, and the patient was discharged home.

After 2 weeks, we removed the lead; the maneuver was easy and uneventful. The patient reported no episodes of urge incontinence: the ICIQ-SF score was 2 out of 19. The patient reported a pain reduction (NRS $=1-2$ out of 10 ). We administered the ICSI ( 3 out of 19) and ICPI (6 out of 16). A bladder diary revealed a reduction in urinary frequency $(12 \rightarrow 5)$ and nycturia $(4 \rightarrow 1)$. The patient had no sexual intercourse in the considered period. The symptom improvement was $>50 \%$, so the patient was recommended to receive a definitive SNM implant.

We recorded no postoperative complications within 1 month.

\section{DISCUSSION}

The current strategy to place the lead for the PNE test has several advantages: low-cost equipment, a rapid procedure, a minimally invasive approach, and safety as outpatient surgery [7]. However, this procedure has significant technical limitations due to the difficulties associated with optimal lead placement along the afferent roots at the medial superior quarter of the S3 foramen, where the best nerve responses are obtained according to the indications by Matzel et al. [8]. Indeed, the insertion may be challenging under only fluoroscopic and/or ultrasound guidance. These limits may be overcome by novel SNSs.

Our case report was a proof of concept for CALP during the PNE test. We reported its feasibility in terms of all the materials and methods described, and-most importantly - its safety. Our approach provided significant assistance during preoperative planning and intraoperative guidance.

Regarding the technical details, EM-guided surgical navigation was used instead of optical tracking, as the emitter detected the stylet tip regardless of tissue deformations during lead insertion [9]. Since the image-to-patient registration was the most important step for accurate surgery, it was optimized by highquality image acquisition and effective placement of the fiducial markers, which allowed a valid REM ( $<2 \mathrm{~mm})$. The patient tracker (i.e., the reference system) was attached to the patient, so body movements did not affect the reliability of the navigation.

The patient reported significant symptom improvement, as objectively measured through NRS and questionnaires reporting $>50 \%$ improvement, which is generally considered the successful endpoint of the PNE test. Therefore, we planned to place a definitive SNM implant in the patient. Thanks to the registered target points, it was possible to place the tined lead in the same position reached during the successful PNE test, whereas it would have been much more difficult with the standard approach.

This case report represents stage 1 of the IDEAL framework for surgical innovations [10]. We presented our technical achievements in detail to favor the adoption of our methodology by other centers. Our experience suggests that the learning curve may be steep, especially because we did not follow a specific course. CALP adoption may be facilitated in hospitals where similar SNSs are available and may be shared. Further cost-benefit analyses should establish whether the increased expenses would be overcome by improved clinical outcomes associated with the potential benefits of CALP (e.g., increased surgical accuracy, facilitation of complex surgery, possibility to replace a lead in the same successful position after a PNE test or in case of lead displacement and/or substitution).

To our knowledge, this is the first study reporting the use of an image-guided SNS for lead placement during the PNE test in a candidate for SNM. CALP is the next step of SNM, as the adoption of SNSs may enable easy and safe lead placement along the $\mathrm{S} 3$ afferent nerve roots.

\section{ACKNOWLEDGEMENTS}

We would like to thank our staff and Drs. Sberna and Lo Russo, chiefs of the Units of Neuroradiology and Epilepsy Surgery at ASST Grande Ospedale Metropolitano Niguarda, for their collaboration.

\section{AUTHOR CONTRIBUTION STATEMENT}
- Conceptualization: $G S$
- Data curation: GS, MS
- Methodology: GS, MS
- Project administration: GS, MS
- Visualization: $G S, E M, M S$
-Writing-original draft: GS 
- Writing-review \& editing: GS, EM, MS

\section{REFERENCES}

1. Mahran A, Baaklini G, Hassani D, Abolella H, Safwat A, Neudecker M, et al. Sacral neuromodulation treating chronic pelvic pain: a meta-analysis and systematic review of the literature. Int Urogynecol J 2019;30:1023-35.

2. Jang HJ, Kwon MJ, Cho KO. Central regulation of micturition and its association with epilepsy. Int Neurourol J 2018;22:2-8.

3. Spinelli M, Giardiello G, Gerber M, Arduini A, Van Den Hombergh U, Malaguti S. New sacral neuromodulation lead for percutaneous implantation using local anesthesia: description and first experience. J Urol 2003;170:1905-7.

4. Gahzi AA, Banakhar MA, Elterman DS, Hassouna M. Radiographic position of the electrode as a predictor of the outcome of InterStim therapy. Int Neurourol J 2017;21:289-94.

5. Vaganée D, Voorham J, Voorham-van der Zalm P, De Wachter S. Needle placement and position of electrical stimulation inside sacral foramen determines pelvic floor electromyographic re- sponse-implications for sacral neuromodulation. Neuromodulation 2019;22:709-15.

6. Cardinale F, Casaceli G, Raneri F, Miller J, Lo Russo G. Implantation of stereoelectroencephalography electrodes: a systematic review. J Clin Neurophysiol 2016;33:490-502.

7. Crites-Bachert M, Clark C. Needle placement: a guide to predictable sensory and motor responses based on variations in needle placement during sacral neuromodulation procedures. Int Neurourol J 2019;23:302-9.

8. Matzel KE, Chartier-Kastler E, Knowles CH, Lehur PA, MuñozDuyos A, Ratto C, et al. Sacral neuromodulation: standardized electrode placement technique. Neuromodulation 2017;20:816-24.

9. Takenaka T, Toyota S, Kuroda H, Kobayashi M, Kumagai T, Mori K, et al. Freehand technique of an electromagnetic navigation system emitter to avoid interference caused by metal neurosurgical instruments. World Neurosurg 2018;118:143-7.

10. McCulloch P, Altman DG, Campbell WB, Flum DR, Glasziou P, Marshall JC, et al. No surgical innovation without evaluation: the IDEAL recommendations. Lancet 2009;374:1105-12. 